\title{
LETTERATURA E Cinema: ANALISI DI UN RAPPORTO
}

Maria Pacella

ABSTRACT L'articolo presenta il rapporto letteratura-cinema come un rapporto istituzionale in continua evoluzione che negli ultimi anni sta assumendo una veste diversa: da una parte non esisterebbero i film senza i libri, visto che l' $80 \%$ dei film deriva da un libro, dall'altra, una volta divenuto film, il libro impallidisce di fronte al bagliore dell'immagine; è vero inoltre che non esisterebbero alcuni libri senza i film visto che dei libri sono influenzati dai film. Esiste dunque un rapporto circolare di grandissima compenetrazione in cui si trapassa da un linguaggio ad un altro, ma da cui emergono anche l'irriducibile diversità e la concorrenza nell'occupare gli spazi individuali e sociali.

PAROLE CHIAVE letteratura; cinema; romanzo; sceneggiatura. 
RESUMO O artigo apresenta a relação entre literatura e cinema como uma relação institucional em contínua evolução que, nos últimos anos, está assumindo uma configuração diferente: de um lado não existiriam fílmes sem os livros, visto que $80 \%$ dos filmes nascem de um livro; de outro, uma vez que se tornou filme, o livro perde brilho diante do esplendor da imagem; é verdade, ainda, que não haveria certos livros sem os filmes, visto que livros são influenciados por filmes. Existe, portanto, uma relação circular de grande compenetração em que se passa de uma linguagem a outra, mas da qual emergem também sua insuperável diversidade e a concorrência na ocupação de espaços individuais e sociais.

PALAVRAS-CHAV literatura; cinema; romance; roteiro.

ABSTRACT This article presents the relationship between literature and cinema as an institutional relationship in continuous evolution, which in past years has begun to change contours: on the one hand, movies would not exist without books, since $80 \%$ of the movies are adapted from books; on the other, once made into a movie, books lose their luster to the splendor of image; it is also true that certain books would not exist without the movies since books are influenced by movies. There is therefore a circular relationship of intense overlap that moves from one language to the other, but from which derive unbeatable diversity and competition to occupy individual and social spaces. KEYWORDS literature; cinema; novel; script. 


\section{Ogginon è più la letteratura ad offrire i racconti di cui ogni società sembra aver bisogno per vivere, bensì il cinema.}

Tzvetan Todorov

E certamente il nostro tempo... preferisce l'immagine alla cosa, la copia all'originale, la rappresentazione

alla realtà, l'apparenza all'essere... Ludwig Feuerbach (Prefazione alla $2^{a}$ edizione de L'essenza del cristianesimo)

L'intera vita delle società, in cui dominano le moderne condizioni di produzione, si annuncia come un immenso accumulo di spettacoli. Tutto ciò che era direttamente vissuto si è allontanato in una rappresentazione. Guy Debord, La società dello spettacolo.

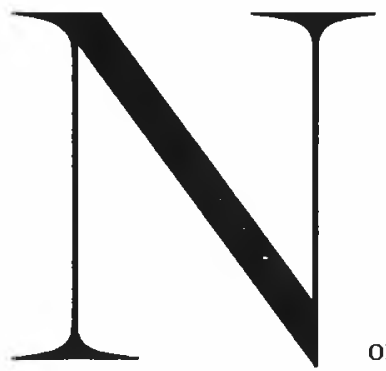

onostante la forte differenza d'età fra letteratura e cinema, quella arte vecchia di secoli, questa vecchia solo di poco più di un secolo, la relazione fra i due ambiti di espressione si è presentata fin dal suo sorgere come un rapporto imprescindibile e insostituibile. Se il cinema nasce come applicazione della fotografia, è pur vero che ha trovato nella letteratura, nelle opere letterarie e negli scrittori i supporti indispensabili con cui crescere e svilupparsi. Diversi nei modi di produzione e fruizione, letteratura e cinema molte volte dalla maggioranza delle persone non vengono vissuti come due ambiti ricchi di scambi e interconnessioni, ma come mondi separati.

Moltissimi film nascono da un libro preesistente ${ }^{\text {. }}$

Negli anni'10 ifilm che si giravano in Italia erano, quasi tutti, attinti da libri letterariantichie moderni, alti ebassi, italianie stranieri. Trail 1910-16 si possono citare, ad esempio, film tratti da Omero, Dante, Tasso, Shakespeare, D’Annunzio, da I Promessi Sposi di Manzoni. Altre volte il cinema attingeva a libri di un livello molto più basso, ai romanzi d’appendice, a Carolina Invernizio ed altri.

1. "Ben 350 degli ultimi 400 film nascono da un romanzo", cosi Angelo Guglielmi ideatore del festival internazionale di cinema e letteratura “Le parole dello schermo", svoltosi a Bologna dal 28/06/2005 al 1/7/2005. Cfr. anche l'articolo online Buio in Sala: il cinema in Biblioteca: "Dal testo al film: passaggio consueto e quanto mai dibattuto, visto che ormai l' $80 \%$ dei film ha alle spalle un libro (anche se non sempre di alto valore artistico) confermando una tendenza nata a cavallo tra le due guerre mondiali... Ultimamente il rapporto si è fatto più stretto, sino a diventare inevitabile. Si può affermare che negli ultimi anni non è uscito libro che avesse un minimo di successo editoriale, senza che questo diventasse, in tempi sempre più rapidi, soggetto per un film." http.//www.concordi.it/img_index7attivita/biblioteca_cinema.htm, accesso del 16/10/2005. Un bestseller riceve la sua consacrazione dal fatto di essere trasformato in film, il che, a sua volta, produce un aumento delle vendite del libro secondo un circolo virtuoso e ricorsivo fra le due arti. Inoltre, secondo un'indagine realizzata da Demaskopea per l'associazione 
Questo ricorso massiccio alla letteratura, a poeti e scrittori celebri o affermati, aveva, certo, anche lo scopo, per il cinema, di ottenere un riconoscimento qualitativo dal punto di vista artistico ed estetico, ma nasceva soprattutto da una necessità.

Il cinema, infatti, scopri ben presto la sua vocazione a narrare storie (dice Christian Metz che "il cinema ha la narrativita strettamente avvinta al corpo"), abbandonando la strada del documentario e del notiziario, e si servi della "vecchia" letteratura come magazzino di racconti da cui estrarre i contenuti per narrare le sue storie ad un pubblico sempre più vasto che aveva "fame di racconto". Il cinema, insomma, doveva rispondere a quel bisogno individuale e sociale di realtà e di fantasia, di sogno e di evasione che è connaturato nell'uomo e che prima era saturato dalle narrazioni scritte o orali. Da qui il grande successo di quest'arte nuova che raccontava attraverso le immagini ${ }^{2}$, essendo cosi accessibile anche agli strati sociali più popolari, e da qui anche la fascinazione e la magia che non accennano a diminuire.

Ma come si comportarono, che reazione ebbero i letterati italiani alla nascita della settima arte ${ }^{3}$ ? Mostrarono molto interesse quasi fin dall'inizio. Le loro reazioni di disprezzo sono in realtà una posizione di difesa delle vecchie e tradizionali arti, anzitutto il teatro - con cui il cinema entrava direttamente in concorrenza - ela letteratura, e rivelano un misto di paura e curiosità, paura di essere superati dal cinema, che ancora non viene recepito come arte, ma anche curiosità per le applicazioni del nuovo mezzo, tanto è vero che molti di loro vi presero parte seppur in incognito. Una reazione, per certi versi, ambigua, ambivalente, a volte ipocrita. Ad esempio, per il poeta e scrittore Guido Gozzano il cinema non potrà mai essere un'arte "come la mosca non diventerà mai farfalla", ma, nello stesso tempo, cercando di non farlo sapere, lavorava per il cinema: nel 1911 aveva steso il commento per un documentario sulla Vita delle farfalle e aveva fornito il soggetto a La storia di Piccolino di Giovanni Vitrotti. Quando mori, stava scrivendo un"“orditura cinematografica": San Francesco. Nel 1913 il critico Renato Serra, pur riconoscendo che il cinema poteva fornire agli scrittori occasioni di maggior guadagno, osservava che "I Magazines (i rotocalchi) stanno alla letteratura press'a poco come il cinema al teatro", con ciò definendola arte decisamente inferiore. Negli anni '10-'20 Verga aveva scritto di nascosto gli adattamenti dei suoi romanzi, attribuendone però la paternità alla sua amante. Al contrario, D’Annunzio, provocatoriamente, nel 1914 firma soggetto, sceneggiatura e didascalie di Cabiria, il film diretto da Pastrone, e nello stesso anno, in un'intervista al Corriere in occasione dell'uscita del film, paragona il cinema alle Metamorfosi di Ovidio per la sua capacità di creare il meraviglioso, elemento fondamentale per un'arte piacevole. Nel contempo scrive al suo editore: "Cabiria è quello che il buon Pascarella [un poeta dialettale romanesco a quei tempi notissimo] chiamerebbe una boiata: è un saggio ironico di arte per la folla arida e melensa”. Un atteggiamento contraddittorio e persino opportunista. Questo disprezzo per il cinema, "arte" popolare e di massa, contrapposto al teatro, vera arte per palati fini, è un atteggiamento comune a molti.

Anche per Piero Gobetti il cinema e il teatro di varietà, conquistando le masse, avrebbero lasciato il teatro al pubblico ristretto degli intenditori. E per Antonio Gramsci c’è una vasta

italiana editori (Aie), in quattro anni, tra il 2000 e maggio 2004, sono usciti in Italia 217 film tratti da libri, di cui 43 sono di autori italiani, circa il $20 \%$ del totale. Dunque la maggior parte degli autori o produttori di cinema italiani preferisce libri stranieri. Attualmente la maggioranza dei film in uscita o in preparazione, in Italia e all'estero, ha origine da un romanzo.

2. "L'immagine cinematografica attinge ad un immaginario collettivo, non individuale. Ė questa la differenza con la parola scritta. La parola è implosione, è personale. Il consumo di cinema è sempre un consumo di tipo sociale". Cosi Renato Candia in http://www. ilcinemante.com/ dettaglio.asp?id=29, accesso del 15/7/2007

3.Il cinema era nato in Italia nel 1896, solo che le prime opere erano documentari di pochi minuti; il primo vero film, sempre di pochi minuti, è La presa di Roma del 1905. 
parte di pubblico che "ha un gran bisogno di divertirsi con una pura e semplice distrazione visiva; il teatro industrializzandosi ha cercato, in questi ultimi tempi, di soddisfare solo questo bisogno. Eil cinematografo, che quest'ufficio può compiere con più agio e più a buon mercato, lo supera nel successo, e tende a sostituirlo"

Ma negli anni'10 la riflessione migliore sul cinema viene dalle riviste di settore su cui poi scrissero anche letterati del tempo come Roberto Bracco, Goffredo Bellonci, Antonio Baldini, Luigi Chiarelli, Lucio d'Ambra ed altri. Nel 1911 Palazzeschi pubblica un romanzo un po' strampalato, Il codice di Perelà, che, nella struttura e nella scrittura, sembra essere influenzato dal cinema. Nel 1916 i futuristi nel loro Manifesto sul cinema parlano della simultaneità come dell'elemento di novità della nuova arte e attaccano il libro, il teatro e l'aspetto letterario del cinema.

Il rapporto di Pirandello con il cinema è un rapporto complesso e ambivalente. Egli comprende subito la profonda diversità del cinema che propone le immagini della realtà ricreate attraverso la macchina in cui gli attori non hanno un rapporto diretto con il pubblico, ma diventano delle immagini in movimento staccate da se stessi e individua nella presenza della macchina che, per la prima volta, si interpone fra il creatore e la sua opera, la novità epistemologica del modo della produzione e del modo della ricezione che richiederebbe quindi altre poetiche e altri parametri interpretativi, non solo dell'arte ma della realtà stessa. È questo che lo spaventa e lo attrae nello stesso tempo, in quel misto di conservatorismo e di innovazione che è cosi caratteristico della sua personalità. Pirandello è perciò tentato dal cinema, ne subisce la fascinazione fin dall'inizio.

Ma vediamo quali furono in concreto le relazioni tra Pirandello e il cinema. All'inizio degli anni '10, dunque agli albori del cinema in Italia, quando vive a Roma e insegna al Magistero, inizia a frequentare i teatri di posa e scrive il soggetto di un film che non sarà mai realizzato. Nel 1915 collabora alla riduzione cinematografica di Le confessioni di un italiano di Ippolito Nievo, anche questo un progetto che non si realizzò mai. Nello stesso anno, però, da queste esperienze nascerà il romanzo Si gira... che, nelle edizioni successive, prenderà il titolo di Quaderni di Serafino Gubbio operatore ${ }^{4}$, tutto ambientato nel mondo del cinema - ed è questa una estrema innovazione che rivela un profondo interesse - quando il cinema non era ancora quel fenomeno di massa che sarà poi. Pirandello tenta di mostrare, e forse anche di esorcizzare, la novità del cinema attraverso una storia esemplare. Questo primo romanzo cinematografico della letteratura moderna ${ }^{5}$ si presenta come un romanzo-diario in cui il protagonista-narratore Serafino Gubbio, operatore in uno stabilimento cinematografico in cui ha il soprannome di "Si gira", descrive e presenta il mondo del cinema anzitutto attraverso la propria autorappresentazione di uomo-macchina, di essere umano totalmente asservito alla macchina, fuso e anzi assorbito da essa, ridotto "a non essere altro che una mano che gira una manovella" Questa vicenda esistenziale tragica - che contiene altre tragedie, l'omicidio e il suicidio di due attori, quasi ad indicare i rischi connessi alla nuova "arte meccanica" - di perdita di umanità, di vitalità, di passioni, introduce alla tragedia storica del trionfo delle macchine, della tecnologia e del mercato, vista come un' irrimediabile

4. Si gira... usci prima, a puntate, sulla rivista Nuova Antologia (giugno-agosto 1915); poi fu raccolto in volume, con lo stesso titolo nel 1916; infine riapparve col titolo definitivo di Quaderni di Serafino Gubbio operatore nel 1925.

5. W. Benjamin, nel suo saggio L'opera d'arte nell'epoca della sua riproducibilità tecnica, Torino, Einaudi, 1966 (testo originale 1936) rende omaggio a Pirandello riconoscendogli "una sorta di primato europeo nella percezione (sia pure al negativo) dei nuovi effetti di questa asportazione (o di questo trasporto fuori di sé) dell'immagine, tra i quali la nascita del 'divismo"' cfr. G. Mazzacurati, Pirandello nel romanzo europeo, Bologna, Il Mulino, 1995, p. 253. 
apocalisse che porterà solo follia e sconvolgimenti con la distruzione del livello naturale. Serafino/macchina, che ha perso la parola per lo choc, è già l'emblema di questa trasformazione, 0 meglio deformazione, il risultato-monstrum dell'effetto delle trasformazioni tecnologiche ${ }^{6}$.

E tuttavia, quasi contraddittoriamente, il romanzo, nel modo stesso in cui è scritto, imita il cinema rubandogli alcune delle tecniche e riproponendole nella sua struttura. Già questo è il segno di un'ambivalenza e di un'attrazione nonostante la narrazione della tragedia del protagonista e la visione apocalittica prospettata, attrazione confermata dal fatto che lo stesso Pirandello nel 1918 propose che il romanzo venisse trasposto in una versione cinematografica che, in realtà, non è stata mai realizzata e che si sta realizzando solo adesso con la realizzazione di un film liberamente tratto da esso ${ }^{7}$

Dal '19 in poi iniziano ad essere tratti film dalle sue novelle. Nel 1924 lo stesso Pirandello scrive una sceneggiatura de $I$ seipersonaggi in cerca d'autore, ma il film non viene realizzato. Poi ci sono alcune sue piccole collaborazioni a sceneggiature. Nel 1930 segue a Hollywood le riprese del film Come tu mi vuoi, tratto dall'omonima commedia e interpretato da Greta Garbo. Negli stessi anni Mussolini, ingelosito dal successo americano di Pirandello e temendo che gli portino via il maggior scrittore italiano, gli chiede "di scrivere un soggetto per un film in glorificazione del lavoro", ambientato nelle Acciaierie di Terni. Pirandello ne scrive il brogliaccio affidandolo poi al figlio per la stesura finale. Il film piacque al regime che si affrettò a realizzarlo attraverso la casa di produzione Cines. Dopo varie peripezie e un tentativo diretto di Pirandello di far realizzare il film al grande Ejzenstejn, che però rifiutò, il film fu realizzato da un regista tedesco che modificò profondamente il progetto pirandelliano. Alla fine il film, col titolo di Acciaio, usci nel 1932 e,pur essendo un buon prodotto, non riscosse molto successo né di pubblico né di critica.

Nel frattempo il cinema nel mondo cresceva e si evolveva tecnologicamente. Il grosso salto era avvenuto nel 1927 con la nascita del primo film sonoro. In I talia bisognerà aspettare il 1930 con un film tratto proprio da una novella di Pirandello. Quest'innovazione aveva aumentato la richiesta di scrittori e drammaturghi specialmente per i dialoghi. In questo modo il cinema sonoro diventava un pericoloso concorrente del teatro. Nel'29 Pirandello scrive un articolo in cui, dauna parte definisce il cinema non vera arte come il teatro, con cui non potrà mai reggere il confronto, dall'altra, cerca di trovargli una collocazione specifica. In questo suo sforzo di allontanarlo dalle arti tradizionali, arriva ad ipotizzare un cinema che fosse "cinemelografia" cioè "linguaggio visibile della musica" un cinema che trasformasse in immagini in movimento i suoni e i ritmi propri della musica ${ }^{8}$.

Nonostante queste teoriche prese di distanza, negli anni '30 lo stesso Pirandello autorizzò gli adattamenti di molte sue opere tra cui, nel ‘37, quello de Il fu Mattia Pascal. Dopo la sua morte, il cinema ha continuato ad attingere a piene mani all'opera pirandelliana che si è offerta, quasi naturalmente, come un immenso corpus di storie da ritrascrivere in chiave cinematografica9

Un rapporto dunque, quello di Pirandello con il cinema, che, anche se non lo vede coinvolto in prima persona, accompagna costantemente la sua produzione letteraria, permettendo 
la ritrascrizione di alcune sue opere. Quello che traspare, al di là delle condanne e delle teorizzazioni, è una posizione di difesa dei mezzi tradizionali di narrazione, il racconto e il teatro, da lui usati e in cui si identifica, ma anche una grande curiosità e partecipazione verso il nuovo mezzo e le sue possibilità espressive.

Dagli anni '20 in poi l'editoria sfrutta il crescente successo di pubblico del cinema e la sua presa sull'immaginario collettivo di massa inserendo le foto di scena dei film nella pubblicazione dei libri da cui quegli stessi film sono tratti e sviluppando un filone d'intrattenimento fatto di romanzi e novelle in cui il cinema compare come uno sfondo fiabesco e esotico.

Negli anni '30-'40 aumentano le collaborazioni di scrittori e critici in campo cinematografico: Giacomo Debenedetti lavora come soggettista, ma anche traduttore-adattatore, di film stranieri, Corrado Alvaro è critico, teorico, soggettista e sceneggiatore, Alberto Savinio fin dagli anni '10 aveva pubblicato scritti sul cinema. Come esempi di film tratti da romanzi negli anni '40 si possono citare: Piccolo mondo antico e Malombra di Mario Soldati tratti da Fogazzaro, Il Marchese di Roccaverdina (da Capuana), Il cappello del prete (da De Marchi), le Sorelle Materassi (da Palazzeschi), tutti di Ferdinando Maria Poggioli. Ossessione di Visconti del 1943 ha un'ascendenza composita: si ispira al romanzo americano Il postino suona sempre due volte di James Cain ed al film Le dernier tournant di Pierre Chenal, un noir francese, riletti alla luce del realismo verghiano e del gusto per il melodramma.

Trail 1945 e il 1948 il neorealismo è sia una corrente cinematografica che letteraria. Tra il' 45 e il' 55 le presenze di scrittori nel cinema sono molte e incisive: Mario Soldati continua come regista (aveva iniziato nel '34) e Cesare Zavattini scrive moltissime e importanti sceneggiature facendo passare in secondo piano la sua attività di scrittore. Altri scrittori che scrivono sceneggiature sono: Ennio Flaiano, anzitutto, ma anche Giorgio Bassani, Alberto Moravia, Giuseppe Marotta, che sono anche critici cinematografici. Per un breve periodo anche Aldo Palazzeschi fu critico cinematografico.

Un discorso a parte merita Pier Paolo Pasolini, poeta romanziere critico giornalista autore di teatro pittore, che approdò al cinema senza sapere nulla di cinema e divenne regista e sceneggiatore realizzando quasi venti film di grande impatto, del tutto inclassificabili. Non ha mai fatto trasposizioni da suoi romanzi, ma da quelli di altri autori, o ha scritto sceneggiature originali.

Insieme a Soldati è stato uno dei pochissimi letterati del ' 900 che sia stato anche regista, non solo sceneggiatore o soggettista. Nella sua produzione si possono individuare varie fasi: quella di Accattone e di altre opere degli anni '60 in cui realizzò e teorizzò poi il cinema-poesia; quella di EdipoRe, in cui è importante l'influsso della pittura attraverso fotogrammi pittorici (i suoi studi con Longhi); l'ultima, quella della Trilogia narratologica (Il Decameron, I racconti di Canterbury, Le mille e una notte) e poi Salò o le 120 giornate di Sodoma, da Sade.

Fino alla fine degli anni ' 60 continua la stagione d'oro del cinema italiano, aumentano le sale e i film prodotti in Italia sono alcune centinaia all'anno; ormai si parla di industria cinematograficae di fenomeno di massa. I guadagni sono molti e, anche per questo, molti letterati e critici letterari,

morte, sono scaduti anche i diritti d'autore. Pirandello diventa patrimonio nazionale, e questo potrà rappresentare un ulteriore incentivo per la produzione di film tratti dalle sue opere. Tra i progetti in cantiere, si può segnalare la nuova versione di Liolà diretta da Gabriele Lavia, attualmente in fase di produzione. 
nonostante esternazioni di disprezzo e difese umanistiche, sono attratti nell'orbita del cinema e sempre più romanzi vengono "tradotti" in film. Solo per fare qualche esempio, dal racconto di Cesare Pavese Tre donne sole, pubblicato nella raccolta La bella estate (1949), Antonioni trae il film Le amiche (1955) e Pietro Germi con il film Un maledetto imbroglio (1959) adatta per il cinema nientemeno che il Pasticciaccio brutto de via Merulana di Carlo Emilio Gadda. Negli anni '60, tra le riduzioni più importanti si possono segnalare: Senilità (1962) di Mauro Bolognini da Italo Svevo; L'isola di Arturo (1962) di Damiano Damiani da Elsa Morante; Agostino (1962) di Bolognini da Moravia; sempre da Moravia Gli indifferenti (1964) di Francesco Maselli; da Leonardo Sciascia Il giorno della civetta (1968) di Damiani.

Negli anni '70, pur già declinando l'epoca gloriosa del cinema italiano, continua l'uso della letteratura per il cinema: nel 1970 esce Metello di Bolognini da Vasco Pratolini; nel 1971 Francesco Rosi con Uomini contro aveva trasposto Un anno sull'altipiano di Emilio Lussu, poi nel 1978 Cristo si è fermato ad Eboli di Carlo Levi; nel 1972 Le avventure di Pinocchio di Carlo Collodi diventano un film televisivo per la regia di Luigi Comencini, regista di cinema; nel 1976 esce L'innocente di Visconti da d'Annunzio; nel 1978 Todo modo di Elio Petri da Sciascia.

Dagli anni ' 80 ad oggi, in una fase in cui l'attività del cinema italiano è stata in netto calo rispetto ai gloriosi anni ' 50 e' 60 , l'incontro fra scrittori e cinema è continuato in un rapporto ormai stabile, ma sembra essere diventato più evidente e rilevante nell'ultimo decennio in cui la visibilità della letteratura è diventata bassissima e viceversa quella del cinema e del mondo che gligira intorno (divi e dive e i loro gossip, festival, ecc.) al tissima. In questo contesto gli scrittori, soprattutto i giovani, cresciuti in una cultura eminentemente cinematografica e televisiva, sono molto attratti dal cinema e, in parte, dalla televisione el'influenza di questi mezzi sulle loro opere è abbastanza visibile. Non che ieri non lo fosse, già Calvino riconosceva l'influenza del cinema sulla sua scrittura, solo che questa era accompagnata e controbilanciata da quella di molti altri scrittori. Adesso, invece, alcuni giovani scrittori sembrano molto più influenzati da film (soprattutto recenti) e serial televisivi americani che da altri libri. Le loro opere sono romanzi basati sull'azione e sull'immagine, sulla visività (spesso usano citazioni di film) e sulla rapidità dei dialoghi, che usano la lingua standard del quotidiano e frequenti onomatopee. Ł̇ soprattutto nella produzione di giovani scrittori come Niccolò Ammaniti, Tiziano Scarpa, Alessandro Baricco, Elena Stancanelli, Laura Pariani, Isabella Santacroce, Francesco Piccolo e anche di altri che operano in internet, che è riscontrabile un immaginario prevalentemente visivo. Come già detto, si tratta di una generazione che è nata e si è formata più col cinema e con la televisione che con il libro e porta traccia di questo nella sensibilità, nel modo di cogliere la realtà e nel modo di esprimerla.

Molto spesso da questi libri, che portano dentro di sé l'impronta del cinema, nasceranno altri film di cui, a volte, saranno sceneggiatori gli stessi scrittori. Oltre a quelli già citati, si possono fare altri nomi, Andrea De Carlo, Massimo Carlotto, E rmanno Cavazzoni, Antonio Tabucchi, Giancarlo De Cataldo, Margaret Mazzantini, Sandro Veronesi, come esempi di scrittori italiani 
contemporanei, di diverse età, che hanno relazioni con il cinema e ormai anche con la televisione (per le mini-serie, cioè film per la televisione).

Tra i film realizzati da loro libri si possono citare: La voce della luna (1990) di Fellini da Cavazzoni; Certi bambini (2000) dei fratelli Antonio e Andrea Frazzi da Diego De Silva che ha anche scritto la sceneggiatura del film; Io non ho paura (2000) di Salvatores da Ammaniti che ha collaborato anche alla sceneggiatura a più mani; Romanzo criminale (2005) di Michele Placido da De Cataldo, Arrivederci amore, ciao (2005) di Michele Soavi da Carlotto. A ottobre 2007 è iniziata la lavorazione del film Un giorno perfetto del regista italo-turco Ferzan Ozpetek, tratto dall'omonimo romanzo di Melania Mazzucco, una delle scrittrici italiane più quotate oggi, formata in letteratura e in cinema, che aveva iniziato la sua carriera proprio come scrittore di soggetti e sceneggiature cinematografiche. Il 2007, inoltre, segna il ritorno d'interesse, ancora non si sa se duraturo o episodico, del cinema alla narrativa dell'Ottocento. Nel novembre 2007 sono usciti, infatti, Rosso Malpelo di Pasquale Scimeca, tratto dall'omonima novella di Verga, e I Vicerè di Roberto Faenza, dal grande romanzo di Federico De Roberto.

Oggi forse l'esigenza di confronto e di reciprocità è più della letteratura che del cinema. Ieri era stato il cinema che aveva manifestato l'esigenza del raffronto con la letteratura per ricavarne l'investitura di cui aveva bisogno. Anche oggi il cinema trasforma ilibri in film. Solo che a volte il film "sostituisce" il libro. Vecchio di più di un secolo, il cinema, trasformatosi in industria cinematografica, occupa uno spazio e gode di una visibilità enormi. La letteratura è invece un'istituzione in crisi che sembra arrancare dietro al cinema trionfante, cercando di rubargli qualche bagliore. Gli attori e i registi diventano immagini smaglianti e modelli di comportamento, gli scrittori sono immagini sbiadite nella società attuale. La forza del cinema è la forza dell'immagine e dello spettacolo industrialmente organizzato nella società di massa. II "vecchio" libro appare superato dalle nuove tecnologie (per ultima quella di internet) ed è costretto a condividere lo spazio con il film ${ }^{10}$, tentando di riproporre il suo valore in una società che sembra poterne fare a meno.

Ma questa è una difesa di parte, umanistica e generazionale, di una letteratura che si immagina stretta in un angolo dal cinema. Per un autore poco più che quarantenne come Francesco Piccolo "nessuna letteratura contemporanea [può] fare a meno dell'immaginario cinematografico nel modo di raccontare, di usare il tempo narrativo e le ellissi, nel modo di utilizzare il montaggio. Il montaggio, in particolare, è una peculiarità della letteratura del Novecento, una delle sue maggiori caratteristiche. Non è possibile prescindere dal cinema: è un gene che entra dentro la letteratura grazie alla sua forza. Non se ne può fare a meno e nessuno ne vuole fare a meno"" .

10. Un segno tangibile della penetrazione del cinema nella letteratura è la presenza dei dvd accanto ai libri nelle biblioteche comunali italiane $\mathrm{e}$ in molte biblioteche pubbliche.

11. Cfr. http://www.railibro.rai.it/interviste.asp?id=231, cit. 